\title{
ROOTS OF UNITY AS QUOTIENTS OF TWO CONJUGATE ALGEBRAIC NUMBERS
}

\author{
Artūras Dubickas
}

Vilnius University, Lithuania

\begin{abstract}
Let $\alpha$ be an algebraic number of degree $d \geqslant 2$ over $\mathbb{Q}$. Suppose for some pairwise coprime positive integers $n_{1}, \ldots, n_{r}$ we have $\operatorname{deg}\left(\alpha^{n_{j}}\right)<d$ for $j=1, \ldots, r$, where $\operatorname{deg}\left(\alpha^{n}\right)=d$ for each positive proper divisor $n$ of $n_{j}$. We prove that then $\varphi\left(n_{1} \ldots n_{r}\right) \leqslant d$, where $\varphi$ stands for the Euler totient function. In particular, if $n_{j}=p_{j}, j=1, \ldots, r$, are any $r$ distinct primes satisfying $\operatorname{deg}\left(\alpha^{p_{j}}\right)<d$, then the inequality $\left(p_{1}-1\right) \cdots\left(p_{r}-1\right) \leqslant d$ holds, and therefore $r \ll \log d / \log \log d$ for $d \geqslant 3$. This bound on $r$ improves that of Dobrowolski $r \leqslant \log d / \log 2$ proved in 1979 and is best possible.
\end{abstract}

\section{INTRODUCTION}

Let $\alpha$ be an algebraic number of degree $d$ with conjugates $\alpha_{1}=$ $\alpha, \alpha_{2}, \ldots, \alpha_{d}$ over $\mathbb{Q}$, and let $n$ be a positive integer. If $D=\operatorname{deg}\left(\alpha^{n}\right)$ then the list $\alpha_{1}^{n}, \alpha_{2}^{n}, \ldots, \alpha_{d}^{n}$ contains each of $D$ conjugates of $\alpha^{n}$ exactly $d / D$ times. In particular, $D=\operatorname{deg}\left(\alpha^{n}\right)<d$ if and only if $\mathbb{Q}\left(\alpha^{n}\right)$ is a proper subfield of $\mathbb{Q}(\alpha)$. For $n \geqslant 2$ and $d \geqslant 2$ this happens precisely when $\alpha^{n}=\alpha_{j}^{n}$ for some $j$ in the range $2 \leqslant j \leqslant d$, so the quotient of two distinct conjugates of $\alpha$ is a root of unity.

Put

$$
U(\alpha):=\left\{n \in \mathbb{N}: \operatorname{deg}\left(\alpha^{n}\right)<d\right\} .
$$

Clearly, the set $U(\alpha)$ is either empty or infinite, since $n \in U(\alpha)$ implies $n \ell \in U(\alpha)$ for each $\ell \in \mathbb{N}$. Let $F(\alpha)$ be a subset of $U(\alpha)$ which is defined as

2010 Mathematics Subject Classification. 11R04, 11R18.

Key words and phrases. Root of unity, conjugate algebraic numbers, degenerate linear recurrence sequence. 
follows:

$$
\begin{aligned}
F(\alpha):=\{n \in \mathbb{N}: & \operatorname{deg}\left(\alpha^{n}\right)<d \text { and } \operatorname{deg}\left(\alpha^{q}\right)=d \\
& \text { for each } q \in \mathbb{N} \text { satisfying } q<n \text { and } q \mid n\} .
\end{aligned}
$$

As we already observed above, $m \in F(\alpha)$ yields $\alpha^{m}=\alpha_{j}^{m}$ for some $j>1$, so that $\alpha / \alpha_{j}=\exp (2 \pi i u / m)$ with $u \in \mathbb{N}$ satisfying $1 \leqslant u<m$ and, by the definition of $F, \operatorname{gcd}(u, m)=1$. In particular, $\operatorname{deg}(\exp (2 \pi i u / m))=\varphi(m)$ does not exceed the number of roots of unity in the field $\mathbb{Q}\left(\alpha_{1}, \ldots, \alpha_{d}\right)$, so that the set $F(\alpha)$ is finite. (Throughout, $\varphi$ stands for Euler's totient function.) Moreover, writing

$$
F(\alpha)=\left\{m_{1}, \ldots, m_{k}\right\},
$$

where, by the definition of $F, m_{i}$ does not divide $m_{j}$ for $i \neq j$, we have

$$
\varphi\left(m_{1}\right)+\cdots+\varphi\left(m_{k}\right) \leqslant d(d-1),
$$

since there are $d(d-1)$ quotients of two distinct conjugates of $\alpha$ and the degree of each quotient which is a root of unity must be $\varphi\left(m_{j}\right)$ for some $j=1, \ldots, k$. By the above, it is easy to see that the set $U(\alpha)$ can be also given in the form

$$
U(\alpha)=\{\ell m: \ell \in \mathbb{N}, m \in F(\alpha)\} .
$$

Various aspects of the sets $U(\alpha), F(\alpha)$ themselves and their complements $\mathbb{N} \backslash U(\alpha), \mathbb{N} \backslash F(\alpha)$, the smallest positive integer $t$ for which the sets $F\left(\alpha^{t}\right), U\left(\alpha^{t}\right)$ are empty, etc. with their applications to linear recurrence sequences and to other problems of number theory have been investigated in [1-6], [7, Chapter 2], [8,11-13]. The relation of the problem to linear recurrence sequences rests on the fact that the sets $F(\alpha), U(\alpha)$ are empty iff the linear recurrence whose characteristic polynomial is the minimal polynomial of $\alpha$ over $\mathbb{Q}$ is nondegenerate.

In particular, one of the results of Dobrowolski in his famous paper [3], where a so far unbeaten estimate for the Mahler measure $M(\alpha)$ of an algebraic integer $\alpha$ which is not a root of unity was obtained, is the following:

Theorem 1.1 (Lemma 3 in [3]). For each $\alpha$ of degree $d \geqslant 2$ the set $U(\alpha)$ contains at most $\log d / \log 2$ prime numbers.

Note that, by (1.1), the prime number $p$ belongs to $U(\alpha)$ if and only if it belongs to $F(\alpha)$. So the same upper bound $\log d / \log 2$ also holds for the number of primes lying in $F(\alpha)$.

Although it is known that the main result of [3] can be obtained without the use of Theorem 1.1, this theorem is of interest itself. A stronger version of Theorem 1.1, although not best possible, was obtained by Matveev (see Lemma 6 and a subsequent remark in [10]). A slightly different proof of Theorem 1.1 is also given in the recent book of Masser [9, Lemma 16.3, p. 204]. 
[9, Exercise 16.6, p. 209] asks whether for $p_{1}, \ldots, p_{r} \in U(\alpha)$, where $p_{1}, \ldots, p_{r}$ are distinct primes, the bound

$$
\left(p_{1}-1\right) \ldots\left(p_{r}-1\right) \leqslant d
$$

is true.

The aim of this note is the next theorem which implies that the inequality (1.2) indeed holds.

Theorem 1.2. Let $\alpha$ be an algebraic number is of degree $d \geqslant 2$. Suppose that the set $F(\alpha)$ contains some pairwise coprime integers $n_{1}, \ldots, n_{r}$. Then,

$$
\varphi\left(n_{1} \ldots n_{r}\right) \leqslant d
$$

In particular, if each $n_{j}=p_{j}, j=1, \ldots, r$, is a prime number, then (1.2) holds, since $\varphi\left(p_{1} \ldots p_{r}\right)=\left(p_{1}-1\right) \ldots\left(p_{r}-1\right)$. To show that the inequality (1.2) is best possible we can consider the number

$$
\beta:=\exp \left(2 \pi i\left(\frac{1}{p_{1}}+\cdots+\frac{1}{p_{r}}\right)\right) .
$$

Then, $\beta$ is a root of unity, $\beta^{p_{1} \ldots p_{r}}=1$ and $p_{1} \ldots p_{r}$ is the smallest positive integer $q$ for which $\beta^{q}=1$. Hence,

$$
d=\operatorname{deg}(\beta)=\varphi\left(p_{1} \ldots p_{r}\right)=\left(p_{1}-1\right) \ldots\left(p_{r}-1\right) .
$$

The conjugates of $\beta$ can be written in the form $\exp \left(2 \pi i\left(k_{1} / p_{1}+\cdots+k_{r} / p_{r}\right)\right)$, where $1 \leqslant k_{j}<p_{j}$ for $j=1, \ldots, r$. Thus, for $\beta$ defined in (1.3), we have $p_{j} \in F(\beta)$ for $j=1, \ldots, r$ (in fact, $F(\beta)=\left\{p_{1}, \ldots, p_{k}\right\}$ ). Hence, we for this $\beta$ we have equality in (1.2).

Note that the left hand side of (1.2) is at least

$$
(2-1) \cdot(3-1) \cdot(5-1) \cdots\left(p_{r}-1\right),
$$

where $p_{r}$ is the $r$ th prime. By the prime number theorem, for this $r$ one has the bound

$$
r \leqslant c \frac{\log d}{\log \log d},
$$

where $d \geqslant 3$ and $c$ is an absolute positive constant independent of $\alpha$ (and so independent of $d$ ). Here, we can take any $c$ greater than 1 for $d$ large enough. The bound (1.4) improves that of Theorem 1.1 and is best possible in the sense that there is an infinite sequence algebraic numbers $\alpha_{k}, k=1,2, \ldots$, such that $\operatorname{deg} \alpha_{k}=d_{k} \rightarrow \infty$ as $k \rightarrow \infty$ for which the number of primes in the set $U\left(\alpha_{k}\right)$ is asymptotic to

$$
\frac{\log d_{k}}{\log \log d_{k}}
$$

as $k \rightarrow \infty$.

In the proof of Theorem 1.2 we shall use the following: 
LEMMA 1.3. If $\alpha$ and $\alpha^{\prime}$ are two conjugate algebraic numbers of degree $d \geqslant 2$ and $\zeta:=\alpha / \alpha^{\prime}$ is a root of unity, then $\operatorname{deg}(\zeta) \leqslant d$.

Various proofs of Lemma 1.3 are given in $[1,4,8,13]$. In the next section we shall prove Theorem 1.2.

\section{Proof of Theorem 1.2}

Let $\mathbb{L}$ be the Galois closure of $\mathbb{Q}(\alpha)$ over $\mathbb{Q}$ and $G:=\operatorname{Gal}(\mathbb{L} / \mathbb{Q})$. Assume that $n_{1}, \ldots, n_{r}$ are pairwise coprime positive integers lying in $F(\alpha)$. Here, $n_{1}, \ldots, n_{r}>1$, since $1 \notin F(\alpha)$. Note that $n_{j} \in F(\alpha)$ yields $\alpha^{n_{j}}=\alpha_{j}^{n_{j}}$, where $\alpha_{j} \neq \alpha$ is a conjugate of $\alpha$ over $\mathbb{Q}$. Furthermore, by the definition of $F(\alpha)$, we have $\alpha^{q} \neq \alpha_{j}^{q}$ for any positive proper divisor $q$ of $n_{j}$. Thus, $\zeta_{j}:=\alpha / \alpha_{j}$ is a root of unity of the form $\zeta_{j}=\exp \left(2 \pi i u_{j} / n_{j}\right)$, where $u_{j} \in \mathbb{N}, 1 \leqslant u_{j}<n_{j}$ and $\operatorname{gcd}\left(u_{j}, n_{j}\right)=1$.

Starting with $\zeta_{1}=\alpha / \alpha_{1}$, we select an automorphism $\sigma_{2} \in G$ which maps $\alpha \mapsto \alpha_{1}$. Applying it to $\zeta_{2}=\alpha / \alpha_{2}$, we find that $\sigma_{2}\left(\zeta_{2}\right)=\alpha_{1} / \sigma_{2}\left(\alpha_{2}\right)$. Multiplying these equalities cancels $\alpha_{1}$, so we obtain

$$
\zeta_{1} \sigma_{2}\left(\zeta_{2}\right)=\frac{\alpha}{\alpha_{1}} \cdot \frac{\alpha_{1}}{\sigma_{2}\left(\alpha_{2}\right)}=\frac{\alpha}{\sigma_{2}\left(\alpha_{2}\right)} .
$$

Next, we select $\sigma_{3} \in G$ which maps $\alpha \mapsto \sigma_{2}\left(\alpha_{2}\right)$ and apply it to $\zeta_{3}=\alpha / \alpha_{3}$. Multiplying (2.1) and $\sigma_{3}\left(\zeta_{3}\right)=\sigma_{2}\left(\alpha_{2}\right) / \sigma_{3}\left(\alpha_{3}\right)$ we further obtain

$$
\zeta_{1} \sigma_{2}\left(\zeta_{2}\right) \sigma_{3}\left(\zeta_{3}\right)=\frac{\alpha}{\sigma_{3}\left(\alpha_{3}\right)} .
$$

Continuing in this way with the next equality $\zeta_{4}=\alpha / \alpha_{4}$, etc. up to $\zeta_{r}=\alpha / \alpha_{r}$ we derive that

$$
\zeta_{1} \sigma_{2}\left(\zeta_{2}\right) \sigma_{3}\left(\zeta_{3}\right) \ldots \sigma_{r}\left(\zeta_{r}\right)=\frac{\alpha}{\sigma_{r}\left(\alpha_{r}\right)}
$$

Since $\zeta_{j} \in \mathbb{L}$ for each $j=2, \ldots, r$, the number $\sigma_{j}\left(\zeta_{j}\right)$ is conjugate to $\zeta_{j}$ for $j=2, \ldots, r$. Hence, $\sigma_{j}\left(\zeta_{j}\right)=\exp \left(2 \pi i w_{j} / n_{j}\right)$ for some $w_{j} \in \mathbb{N}$ satisfying $1 \leqslant w_{j}<n_{j}, \operatorname{gcd}\left(w_{j}, n_{j}\right)=1$. Setting, for simplicity of notation, $w_{1}:=u_{1}$ we find that the left hand side of $(2.2)$ is equal to

$$
\zeta=\exp \left(\frac{2 \pi i w_{1}}{n_{1}}\right) \prod_{j=2}^{r} \exp \left(\frac{2 \pi i w_{j}}{n_{j}}\right)=\exp \left(2 \pi i\left(\frac{w_{1}}{n_{1}}+\cdots+\frac{w_{r}}{n_{r}}\right)\right) .
$$

Since $\zeta$ is a root of unity and, by (2.2) and (2.3), equals the quotient $\alpha / \sigma_{r}\left(\alpha_{r}\right)$ of two conjugates of $\alpha$ of degree $d$, from Lemma 1.3 we deduce that

$$
\operatorname{deg}(\zeta) \leqslant d
$$

Consider the number

$$
\frac{w_{1}}{n_{1}}+\cdots+\frac{w_{r}}{n_{r}}=\frac{w}{n_{1} \ldots n_{r}},
$$


where $w:=\sum_{i=1}^{r} w_{i} k_{i}$ and $k_{i}:=\prod_{j \neq i} n_{j}$. We claim that $\operatorname{gcd}\left(w, n_{1} \ldots n_{r}\right)=$ 1. Indeed, for a contradiction suppose that there is a prime number $p$ which divides $n_{1} \ldots n_{r}$ and $w$. Without restriction of generality we can assume that $p \mid n_{1}$. Then, using $p \mid k_{i}$ for $i=2, \ldots, r$ and $p \mid w$, we deduce that $p \mid w_{1} k_{1}$. However, in view of $\operatorname{gcd}\left(w_{1}, n_{1}\right)=1$ and $p \mid n_{1}$ the number $p$ does not divide $w_{1}$. Similarly, $p$ does not divide $k_{1}=n_{2} \ldots n_{r}$, since for each $j \geqslant 2$ the numbers $n_{j}$ and $n_{1}$ are coprime.

Now, from (2.3) and (2.5), it follows that

$$
\zeta=\exp \left(2 \pi i w /\left(n_{1} \ldots n_{r}\right)\right)
$$

where $w \in \mathbb{N}$ and $\operatorname{gcd}\left(w, n_{1} \ldots n_{r}\right)=1$. Consequently, $\zeta^{n_{1} \ldots n_{r}}=1$, where $n_{1} \ldots n_{r}$ is the smallest positive integer with this property. Hence, $\operatorname{deg}(\zeta)=$ $\varphi\left(n_{1} \ldots n_{r}\right)$ and so $(2.4)$ implies the required inequality $\varphi\left(n_{1} \ldots n_{r}\right) \leqslant d$.

ACKNOWLEDGEMENTS.

I thank the referees for suggesting some simplifications and other useful remarks and corrections.

\section{REFERENCES}

[1] M. G. Aschbacher and R. M. Guralnick, On Abelian quotients of primitive groups, Proc. Amer. Math. Soc. 107 (1989), 89-95.

[2] J. Berstel and M. Mignotte, Deux propriétés décidables des suites recurrentes linéaires, Bull. Soc. Math. France 104 (1976), 175-194.

[3] E. Dobrowolski, On a question of Lehmer and the number of irreducible factors of a polynomial, Acta Arith. 34 (1979) 391-401.

[4] P. Drungilas and A. Dubickas, On subfields of a field generated by two conjugate algebraic numbers, Proc. Edinburgh Math. Soc. 47 (2004), 119-123.

[5] A. Dubickas, Roots of unity as quotients of two roots of a polynomial, J. Austral. Math. Soc. 92 (2012), 137-144.

[6] A. Dubickas and M. Sha, Counting degenerate polynomials of fixed degree and bounded height, Monatsh. Math. 177 (2015), 517-537.

[7] G. Everest, A. van der Poorten, I. Shparlinski and T. Ward, Recurrence sequences, Mathematical Surveys and Monographs 104, American Mathematical Society, Providence, RI, 2003.

[8] I. M. Isaacs, Quotients which are roots of unity (solution of problem 6523), Amer. Math. Monthly 95 (1988), 561-562.

[9] D. Masser, Auxiliary polynomials in number theory, Cambridge Tracts in Mathematics 207, Cambridge University Press, Cambridge, 2016.

[10] E. M. Matveev, On a connection between the Mahler measure and the discriminant of algebraic numbers, Math. Notes 59 (1996), 293-297.

[11] P. Robba, Zéros de suites récurrentes linéaires, Groupe Étude Anal. Ultramétrique, 5e Année (1977/78), Exposé No. 13, Paris, 1978, 5 p.

[12] A. Schinzel, Around Pólya's theorem on the set of prime divisors of a linear recurrence, in: Diophantine equations. Tata Inst. Fund. Res. Stud. Math., 20, Tata Inst. Fund. Res., Mumbai, 2008, pp. 225-233.

[13] K. Yokoyama, Z. Li and I. Nemes, Finding roots of unity among quotients of the roots of an integral polynomial, in: Proceedings of the 1995 international symposium on symbolic and algebraic computation (ed. A.H.M. Levelt), ISSAC'95, Montreal, Canada, July 10-12, 1995, New York, NY: ACM Press, 1995, pp. 85-89. 
A. Dubickas

Department of Mathematics and Informatics

Vilnius University

Naugarduko 24, Vilnius LT-03225

Lithuania

E-mail: arturas.dubickas@mif.vu.lt

Received: 13.4.2017.

Revised: 29.5 .2017$. 